\title{
CONDICIONES EPISTÉMICAS Y METAMETODOLÓGICAS DE LA INVESTIGACIÓN EN COMUNICACIÓN *
}

EPISTEMIC AND META METHODOLOGICAL OF RESEARCH IN COMMUNICATION CONDITIONS

\section{[ CARLOS DEL VALLE ROJAS ]}

Periodista, Licenciado y Magister en Comunicación, Universidad de La Frontera, Chile. Doctor en

Comunicación de la Universidad de Sevilla, España. Posdoctorado en Estudios Culturales por la Universidad Federal de Río de Janeiro, Brasil. Investiga en comunicación, educación e interculturalidad. Es autor y co-autor de más de 130 publicaciones. Investigador acreditado en CONICYT de Chile, CNPq de Brasil y SICA de España. Decano de la Facultad de Educación, Ciencias Sociales y Humanidades, Universidad de La Frontera. Email: carlos.delvalle@ufrontera.cl

Recibido: octubre 27 de 2014

Aceptado: noviembre 13 de 2014
* El presente trabajo corresponde a la transcripción literal de un taller dictado por el autor en el Instituto de la Comunicación e Imagen de la Universidad de Chile. El autor agradece el trabajo de transcripción y organización del texto del Dr. Claudio Salinas, académico de dicho Instituto. 
Hablar sobre las metodologías de investigación resulta amplio y pretencioso, porque vamos a hablar de un tipo de metodologías que corresponden a determinadas técnicas que se trabajan en el campo de la investigación en comunicación. En este sentido, la metodología también tiene mucho de estrategia, no solamente una presentación de técnicas o dispositivos de análisis, sino también una estrategia que cada investigador utiliza para poder relacionarse con el objeto-corpus o con cualquier objeto/sujeto que se esté analizando. En esa misma dirección, es importante preguntarse en qué medida las metodologías que uno está trabajando atienden de buena manera un objeto de estudio, puesto que este es una construcción teórica.

Palabras Clave: Metodología, investigación, comunicación, condiciones.

\section{ABSTRACT}

Talking about methodologies of research is broad and pretentious, because we are going to talk about methodologies that correspond to certain techniques used in the field of research in communication. The methodology in this regard also has a lot of strategy, not just a presentation of techniques or devices, but also a strategy used by each researcher to be able to interact with the object-corpus or any object/subject that is parsed. In the same direction, it is important to ask ourselves to what extent the methodologies one is working with attend in a good way to an object of study, since that object is a theoretical construct.

Keywords: Methodology, research, communication, conditions.

\section{PRESENTACIÓN}

Espero que este texto cumpla con el objetivo de ser práctico, aunque eso dependerá del trabajo posterior del lector, puesto que por mi parte sólo puedo garantizar la exposición de ideas y aportes.

Así entonces, la metodología asume algunos desafíos que no son menores. Para comenzar, diremos que una metodología debe ser ad hoc, es decir, pertinente a un tipo determinado de construcción teórica que hemos producido, porque si no nos sirve para atender esa construcción teórica, va a ser muy limitada y desde esa limitación no podremos obtener los resultados que queremos. Otro desafío que se asume corresponde a la visualización de la metodología como una decisión que un investigador o conjunto de investigadores toma y, parafraseando a Jameson (1989), esto nos pondría frente a una propuesta ideológica. Lo que quiero señalar es que tampoco está ausente, en la construcción de una estrategia metodológica de una investigación, la respectiva dimensión ideológica, ya que -y permítanme ser claro en este punto- cuando hablamos del acto metodológico, estamos hablando también de un acto ideológico, pues no da lo mismo el tipo de metodologías que estoy usando y mucho menos da lo mismo el tipo de preguntas metodológicas formuladas, eso por un lado.

Por otro, las preguntas que uno se hace como investigador deben centrarse en resolver el problema de fondo ¿las estrategias metodológicas utilizadas están ayudando a resolver nuestras preguntas de investigación? Sin embargo, las preguntas de investigación son cuestiones que no están relacionadas con el plano metodológico sino epistémico, por lo tanto, lo que viene ahora no es un desafío metodológico estricto, sino epistémico, porque cuando estamos hablando del campo de la comunicación, hablamos de la configuración de los saberes que constituyen ese campo y esa es una pregunta epistémica. En cierto sentido, cuando se habla de teoría de la investigación, se refiere a cómo respondemos a las preguntas que nos estamos formulando en el campo de la comunicación y que vienen y devienen de una discusión de tipo epistémica, por eso es que en algunos casos $-y$ no pocos-, las preguntas vienen desde una construcción teórica determinada muy fuerte y lo que estamos haciendo ahora y presenciando hoy día, son procesos de ajustes de cómo las metodologías y técnicas que tenemos, desde las estrategias que utilizamos, nos permiten abordar las nuevas preguntas o las preguntas antiguas que se están reformulando en el campo de la comunicación. Estoy pensando puntualmente en los espacios de discusión y diálogo productivo, dentro del desarrollo teórico de la economía política de la comunicación y la cultura, visto este último como un planteamiento epistémico fuerte, replanteado desde mediados de los noventa, que se ha instalado en la comunicación y tiene toda una serie de connotaciones, digamos parafraseando a Bourdieu (2003), dentro de un campo de batalla. 
Además, debemos pensar que lo anterior nos presenta toda una lógica de los procesos de instalación, en relación con este nuevo conjunto de saberes relacionados con la economía política de la comunicación en un espacio que está en proceso de redefinición. Entonces cuando uno entra en esa línea de trabajo, se formula una pregunta que no se centra en la formas cómo resuelvo lo que ya tengo definido desde las metodologías existentes, sino que nos focalizamos en la interrogante: ¿cómo resuelvo o ayudo a resolver aquellas nuevas preguntas de investigación, mediante la construcción y aplicación de una metodología predeterminada o legitimada por el campo de saberes y la comunidad científica? Planteo esta idea, en vista de que la economía política de la comunicación y la cultura centra sus esfuerzos en elaborar otro tipo de preguntas que son distintas a las que se formula, por ejemplo, la teoría del discurso, la semiótica o la teoría de sistemas. Las preguntas que quiere instalar la economía política de la comunicación y la cultura tienen una carga muy distinta y no es de extrañar, entonces, que las preguntas formuladas en la actualidad, sean interrogantes que cuestionan la forma cómo las metodologías que conocemos y utilizamos puedan resolver nuevas problemáticas de investigación que están surgiendo en distintas partes del mundo.

\section{EL SENTIDO DE LA METODOLOGÍA}

Por consiguiente, una metodología tiene que ser entendida en la medida que pueda ayudarnos a resolver las grandes preguntas que debemos resolver en los estudios de la comunicación. En este preciso momento, quisiera hacer hincapié en que la cuestión a la que nos enfrentamos, además de lo anteriormente expresado, es definir cuáles son las grandes preguntas que tenemos en comunicación. Y esa meta-pregunta tiene que ver con los objetos de estudio que estamos construyendo, aunque da la impresión de que este tipo de discurso se ha instalado en nuestro ámbito como un gran mecanismo cognitivo organizativo que busca plantear y desarrollar objetos de estudio e incluso construir corpus específicos. Desde nuestra perspectiva, podemos asumir que se evidencia un buen avance, es decir, tenemos un recorrido que se visualiza bastante resuelto, hay bibliografía y experiencias que ustedes pueden encontrar. No obstante, aún así me inquietan las nuevas preguntas que podemos responder en el marco de la investigación en comunicación.

Si bien he preparado una presentación general, básicamente por el tiempo que tenemos, y para que dentro de las pretensiones que determinan este espacio de diálogo podamos desarrollar algunos temas centrales que nos reúnen hoy, debemos comenzar a discutir y tener claro que la cuestión epistemológica es un factor clave de la investigación. Si no instalamos la discusión acerca de lo epistemológico, vamos a pensar que estamos hablando sólo de cuestiones procedimentales; de ver cómo se resuelve un cierto objeto o corpus, a partir de un dispositivo que puede ser llevado al extremo como es el caso del uso de un software específico. Para este caso hay un trabajo muy interesante de José Luis Piñuel (2002), publicado en la revista de Sociolingüística de España, la cual describe los más relevantes dispositivos que tenemos desde el punto de vista técnico para hacer análisis de contenido.

En tal caso, no podemos preguntarnos sólo desde una perspectiva técnica o procedimental, la cuestión de la investigación en comunicación. Esta situación nos llevaría a un camino donde solamente vamos a llegar a la entrega de un recetario que contendrá los pasos, procesos, etapas y preguntas a seguir en el desarrollo de una investigación. Dicho de otra forma, es mejor que yo les entregue algunas direcciones en la web para que ustedes puedan utilizar un software de análisis e ingresar allí algunos datos, procesarlos, obtener resultados y después hacer un análisis interpretativo, lo que en términos generales corresponde a la forma en que se está avanzando en muchos ámbitos de la investigación en comunicación. Quizás me atrevería a decir que estamos frente a una injerencia, no menos importante del análisis lingüístico en nuestro campo. En definitiva, la pregunta sigue siendo relevante, en torno a si es conveniente seguir la orientación establecida por la lingüística hoy día, lo que se traduce en una guía hacia el análisis de corpus para dar cuenta de, por ejemplo, tipologías discursivas, o, por el contrario, nos planteamos nuevos retos y desafíos incorporando nuevas preguntas y enfoques teórico-metodológicos para estudiar los fenómenos comunicacionales.

En todo caso, insisto que la pregunta tiene que centrarse en el lugar donde queremos llegar desde el punto de vista epistémico. Me explico, si se logra desde los saberes que quiero develar, explicar, instalar, entonces la estrategia que quiero asumir tiene que ser distinta, por cuanto surge una cuestión inseparable, casi como la economía política. Aquí una cuestión importante es que la metodología no la podemos separar de la teoría, porque la metodología surge de alguna manera con más o menos claridad, con más o menos-diríamos- explicitación de algún marco teórico, aunque si somos realistas y justos, lo que ocurre en la actualidad es que, en muchos casos, hemos avanzado de una manera tal que no reconocemos los marcos teóricos utilizados para determinadas propuestas metodológicas, por lo tanto, con este vínculo estrecho que planteo acerca de lo teórico y metodológico, cobra más sentido y más fuerza sobre todo si consideramos lo que comentaba al comienzo de este taller-, la idea de que todo ejercicio metodológico es un ejercicio ideológico en el sentido de Jameson (1989). 


\section{METODOLOGÍA, CAMPO Y DISCIPLINARIZACIÓN}

Si continuamos ahondando en los temas de este taller, nos daremos cuenta que es evidente el surgimiento de algunas nociones que para mí dan mucho sentido a toda conversación académica. Es probable que hayan aquí asistentes que estén inscritos en el curso etnográfico y van a tener que digerirlo una vez más, pero en el fondo es esto importante. Así es cómo nosotros encaramos una gran cuestión que está detrás del asunto metodológico y es la cuestión de la instalación disciplinaria o lo que se llama, desde la discusión de la epistemología, proceso de disciplinarización del campo de la comunicación. Es decir, ese vínculo de la condición disciplinaria nos ayuda a explicar el porqué tenemos lo que tenemos como estrategias metodológicas instaladas, en tal sentido, se observa que hay disciplinas -en esta noción Bourdiana del campo de conocimiento como un campo de batalla- que han logrado prender con más fuerza y han instalado dispositivos de análisis mayormente utilizados por los distintos investigadores de la comunicación. Así entonces tenemos, por ejemplo, una fuerte influencia de la psicología social, donde vemos que muchas de las investigaciones que nosotros hacemos llevan por título representación social de..., o sea detrás de eso hay toda una corriente no sólo metodológica, sino que teórica, lo que ha dejado de lado a una línea de trabajo en comunicación que se veía a mediados de los noventa con un gran impacto y que era todo lo concerniente a la teoría de la construcción social de la realidad. En el fondo, nuevamente me atrevo a afirmar que, en este caso, el desplazamiento de un enfoque teórico-metodológico, como es el de la teoría de la construcción social de la realidad -a mi juicio-, pasó porque la corriente asentada en el campo de la psicología se fortaleció a partir del desarrollo de la disciplina que la gesta en su matriz de saberes y disciplinamiento para la construcción de sus dispositivos; al contrario de lo que ocurre en la sociología. Son prácticas distintas en dos disciplinas consolidadas. En consecuencia, si observamos los cambios y transformaciones ocurridas en las ciencias sociales, podemos evidenciar que en muchas de nuestras investigaciones estamos hablando de la representación social de tal cosa o de otra, a modo de ejemplo, pero esta situación también ocurre con la teoría del discurso.

Ahora bien, para ir avanzando en algunos puntos, quisiera profundizar acerca de la importancia de la noción de ideología en la investigación en comunicación. Lo que quiero decir es que debemos asumir que la ideología corresponde a una noción que siempre está vinculada y va a dar lugar a la pregunta del porqué estamos usando determinadas estrategias metodológicas para desarrollar la investigación en comunicación. En este mismo sentido, creo oportuno precisar que dentro de la discusión que se debe hacer, en torno al tema de la ideología, no podemos obviar primero la importancia de la historicidad como elemento clave dentro del campo de la comunicación, puesto que cada recorrido o camino de desarrollo histórico de cada uno de los países y territorios de América Latina es distinta. Es más, durante la jornada de ayer sostuve una conversación con Eliseo Verón, donde tuve la oportunidad de consultarle sobre el debate que había a fines de los sesenta entre él y Armand Mattelart cuando estaba en Chile, dicho debate giró en torno a la forma de hacer la investigación en comunicación, donde la propuesta de los interlocutores era si se hace investigación desde una perspectiva ligada al proyecto semiótico que se vinculaba más con procedimientos de tipo científico o, en cambio, si se debía realizar la investigación en comunicación desde un enfoque más político-social, en la medida que el contexto de la época asentaba una preocupación mayor por la ciudadanía, el cambio social y la política de Estado. No obstante, Verón me dio algunas pistas para encontrar en una revista todo un registro acerca de la crítica al proyecto de Mattelart en Chile, lo que sería interesante encontrar porque me parece que la tensión que observamos, en la actualidad, es muy similar a la vivida en esa época, aunque no en el sentido del contexto político, sino más bien en la disputa dentro del campo de investigación.

De aquí, la necesidad de entender que no hemos superado aún este gran dilema, es decir, si nos vamos a la rigurosa cientificidad de los procedimientos o, por el contrario, vamos a la posición más política de la investigación y por ende, entendemos la investigación en comunicación como muchos quieren entenderla: como una investigación orientada al cambio social y a la transformación social, lo que inevitablemente obliga a realizar otras preguntas y plantear otras estrategias metodológicas.

Sin embargo, no podemos obviar que, por un lado, en el área más científica de la investigación en comunicación habrá una serie de estrategias que vienen heredadas desde la lingüística y que nos han determinado la forma en que debemos hacer metodología, tratando de identificar con mucha claridad los elementos categoriales, analíticos, establecer las frecuencias de ocurrencia y después sacar los resultados e interpretaciones; mientras que, por otro, la otra línea más ideológica no busca lo anterior, sino que comienza por la posición que tiene el investigador sobre ese tema para después dar paso a la acción de interpretación e interpelación, es decir, el investigador debe interrogar al objeto de estudio que ha construido desde esa posición, en cuyo caso, también se interroga a sí mismo. Comprenderán ustedes que el debate aquel de fines de los sesenta, principios de los setenta sigue siendo tan vigente hoy -a mi juicio- y los intentos de la economía política de la comunicación y la cultura en la actualidad apuntan a fortalecer una posición más política que científica necesariamente y únicamente. Para 
reflejar tal situación, voy a dar un ejemplo del gran esfuerzo que está haciendo la gente que estudia economía política de la comunicación y la cultura para instalarse en los espacios de poder ligados a la dirección de ALAIC, que corresponde a la asociación que tenemos en Latinoamérica para investigadores de la comunicación. Digamos entonces que la gente que hace economía política de la comunicación y la cultura se ha propuesto asumir la dirección de ALAIC, porque hay un proyecto ideológico y, seamos claros en esto, investigadores de la economía política, Bolaño entre otros, quieren decir desde la plataforma visible de ALAIC: hagamos estudios con compromiso ideológico y recuperemos los grandes proyectos de la comunicación, que no son distintos de los proyectos que tenía la economía política convencional, en el marco de los problemas que surgen desde las culturas del trabajo en la comunicación y también vincularnos - como decía Veron-con los temas de las profesiones, las culturas del trabajo, las transformaciones de la cultura del trabajo, las relaciones salariales y del tipo de empleo que existe. Lo expuesto, y que he conversado mucho con César Bolaño, serán las mismas interrogantes ligadas a la subsunción del trabajo intelectual al capital.

\section{INVESTIGACIÓN EN COMUNICACIÓN, INSTITUCIONALIZACIÓN Y LEGITIMACIÓN}

Si podemos ampliar un poco más las ideas que hemos enunciado en torno al tema central del taller que nos reúne, quisiera hacer mención al proceso de institucionalización, puesto que no podemos olvidar que la instalación de los estudios de la comunicación -como campo epistémico y metodológico- corresponde o es fruto de un proceso de cristalización que viene y deviene desde las escuelas de formación profesional. Es decir, que primero se enseñaba la formación profesional del periodista y luego surgieron las interrogantes atingentes al desarrollo del campo disciplinario. Tal situación no ocurre, por ejemplo, con la sociología vista como un campo de saberes que permite el surgimiento de las interrogantes sobre los procesos sociales, para después dar paso a la preocupación sobre la formación profesional de sujetos que puedan atender las necesidades de estudio de los procesos sociales respectivos.

En un sentido estratégico, entonces, conocer los procesos de institucionalización de un campo de saberes es un asunto de suma importancia. Se necesita de una capacidad para reconocer el lugar desde donde se está haciendo la investigación, que en nuestro caso -fundamentalmente- fueron y siguen siendo los programas de formación profesional los responsables de su gestación y actual desarrollo. Sin embargo, no debemos olvidar que hay antecedentes que nos permiten asegurar que dicha lógica no es siempre la mejor o la única alternativa, puesto que si recordamos lo que ocurrió con la Escuela de Frankfurt es evidente que la producción de conocimiento se logra desde centros de investigación, como fue el caso del Centro de Investigaciones Sociales en Frankfurt. Por lo tanto, al comprender este proceso de institucionalización, es posible obtener ciertos indicios fundamentales para comprender la importancia de hacer cierto tipo de preguntas y, a su vez, nos facilita la acción de reflexionar sobre lo que estamos haciendo en los trabajos de investigación, acerca de las metodologías que estamos usando $y$, finalmente, preguntarnos acerca de la cientificidad de lo realizado. Esto último, nos lleva de forma inmediata a recordar las dos objeciones que instalan otras disciplinas para decirnos que nosotros somos poco científicos y ustedes las deben haber escuchado en más de alguna oportunidad:

1.Ustedes no tienen marcos teóricos propios, todos sus marcos teóricos utilizados son prestados de la sociología, de la economía, etcétera.

2.Ustedes no tienen metodologías propias.

Respecto a lo primero, quisiera preguntar ¿es relevante pensar en la propiedad de los marcos teóricos utilizados? Puesto que, en estricto rigor, alguien pudiera pensar que es algo relevante, y quizás muchos pretendan utilizar el tiempo académico en debatir sobre la propiedad. Ahora, tampoco vamos a pensar que nuestro campo es absolutamente sólo receptor de enfoques teóricos, porque recordamos que tenemos una influencia fuerte en ciertos marcos teóricos, ejemplifico con una pregunta: ¿qué sería de los estudios de la semiótica sin los avances que ha habido a través de los análisis del objeto de estudio de la comunicación? Incluso podríamos pensar que, en algunos casos, tal respuesta tampoco resulte del todo satisfactoria.

En relación con lo segundo, existe una mayor claridad en reconocer lo que se utiliza y que ha sido producido con anterioridad por otras disciplinas, puesto que trabajamos con algunas estrategias metodológicas, como es el caso de los análisis críticos del discurso, análisis lingüístico. Trabajamos con otras técnicas para la recopilación de información que son prestadas de las ciencias sociales como el grupo de discusión, entrevistas en profundidad, entre otras.

Grosso modo y si analizan los textos clásicos que hay del campo de la comunicación, estoy pensando en textos como "La producción social de la comunicación" de Manuel Martín Serrano (2004), que se ha usado mucho porque tiene una particularidad. Ese texto te ofrece el marco teórico, las categorías conceptuales y después te las transforma en categorías analíticas y te brinda modelos -los modelos canónicos de análisis estructural y cognitivo del relato de los medios de comunicación-. Lo que me llama la atención de ese texto es que para justificar el carácter científico del libro, una de las respuestas que entrega Martín Serrano 
(2004) es que él ha incorporado un elemento que le parece central, desde el punto de vista de la comunidad científica, y que sería el análisis lógico-estadístico.

Siguiendo con el ejemplo de Martín Serrano (2004), podemos ver cómo nosotros tenemos entre manos un problema que gira en torno a la pregunta de cómo logramos mayor cientificidad, lo que evidencia un tipo de interrogante que se nos impone desde las ciencias de la naturaleza y algunas ciencias de la cultura y nos exigen siempre lo mismo: lo cuantitativo por sobre lo cualitativo. Incluso, si nos remitimos a los congresos del campo de la comunicación, se logra evidenciar la instalación en los últimos años, por ejemplo, de un grupo de trabajo denominado "metodología de la investigación en comunicación” en ALAIC y en FELAFACS. Es más, en el Congreso de ULEPICC, que corresponde a un lugar de encuentro de la economía política, apareció ahora la mesa sobre "metodología en investigación en economía política de la comunicación”, es decir varios grupos están preocupados acerca del cómo nos hacemos legítimos y válidos en la comunidad científica que es bastante estricta con nosotros.

Ahora bien, si podemos continuar explayándonos acerca de las diversas aristas que enfrentamos los investigadores y estudiantes, vemos que cuando nos enfrentamos con algunas preguntas de investigación surgen de forma inmediata distintas propuestas acerca, por ejemplo, de la forma cómo desarrollar una investigación de tesis e, incluso, dichas discusiones que se tienen en el seno de un grupo de investigación se observan o están presentes en el marco de la formulación de un proyecto, puesto que todo lo anterior está inserto en un proceso decisional fundamental y, por tal razón, afloran algunas preguntas básicas dentro de todo proceso investigativo: ¿qué hago?, ¿cuál es la problemática a estudiar?, ¿qué enfoque epistemológico, teórico y metodológico he de utilizar?, ¿cuánto tiempo debo establecer para el desarrollo de la investigación?, entre otras variedades de interrogantes que existen.

Pues bien, una primera línea de batalla que enfrenta todo investigador se focaliza en la preocupación acerca del fenómeno de estudio, lo que -en términos generales- es un problema que tiene cabida dentro de la cuestión metodológica, porque para definir un objeto de estudio, debo formular una pregunta teórica, pero con pretensiones de ser analizada, entonces esta siempre va a girar en torno a ¿cómo observaré mi objeto de estudio? No voy a decir cómo lo mido porque corresponde a otro paradigma. Es entonces cómo debemos tener claridad en que todo cambio de perspectiva trae consigo un cambio de opción, en el fondo es una elección -incluso muchas veces arbitraria-, pero donde lo importante es la definición de un objeto de estudio, ser capaz de avanzar desde un tema o idea a un problema de investigación. Es más, la problematización respectiva de mi propuesta viene y deviene a partir de posiciones epistémicas claras.

\section{PERSPECTIVAS METODOLÓGICAS DEL CAMPO DE LA COMUNICACIÓN}

Sin ánimo de caer en una visión limitada de nuestra realidad en el campo de la comunicación, creo que lo descrito en estos minutos, en los que ha transcurrido esta exposición, es el reflejo de lo complejo del problema de la investigación en comunicación. Estamos enfrentados a decisiones relevantes y que marcan el desarrollo de nuestro campo, incluso podemos intentar resolver la primera etapa de una investigación siguiendo las modas teóricas, los objetos de estudio de mayor interés para el mundo académico, pero -y aquí quiero ser claro- siempre vamos a llegar a una dimensión metodológica que, en mi caso, valoro como clave. En este punto, me refiero al tema metodológico, es decir, el repertorio que se propone utilizar va a darnos las posibilidades de desarrollo factible de una investigación, de modo que una pregunta clave es saber ¿qué repertorio metodológico estamos poniendo a disposición? Puesto que si estamos entregando un repertorio metodológico reducido, es obvio que las posibilidades van a ser reducidas. Es más, no debemos extrañarnos de que la mayoría de las investigaciones sean sobre análisis de discursos, análisis de representaciones sociales o algún tipo de análisis semiótico-discursivo. Sin embargo, ¿qué pasa con las metodologías participativas y de enfoque crítico? Esta es una pregunta que guarda una estricta relación con el desarrollo de la economía política de la comunicación y la cultura en nuestro país, ya que no se han logrado establecer con precisión algunas estrategias metodológicas propias que se han de utilizar en el marco de esta perspectiva teórica y sus objetos de estudio de interés. Es tal la importancia que le asigno a este punto del debate que he tratado de establecer diversos diálogos con académicos en distintas instancias, congresos, reuniones, entre otros: ¿podría convivir el análisis del discurso con la economía política?, ¿de qué forma estamos desarrollando investigación en Chile desde una perspectiva crítica?

Veamos otra arista que me interesa mencionar brevemente, a modo de complementar todo lo dicho hasta aquí. En Chile, observamos un fenómeno interesante en términos cuantitativos y que ustedes pueden identificar si rastrean los proyectos FONDECYT asignados durante el 2008, donde se aumentó en un $100 \%$ el número de proyectos de comunicación aprobados en comparación con el 2007. Lo interesante a observar es que dicho aumento de los proyectos de investigación en comunicación se da en el marco de un 
proceso crucial e histórico, donde los estudios de comunicación más han crecido y que corresponde a una etapa donde la comunidad se ha comenzado a interrogar, discutir, dialogary trabajar en torno a la pregunta ¿cómo vamos a investigar algo evitando la repetición de modelos heredados o la aplicación de los mismos esquemas que hemos venido trabajando, pero que están adscritos a otras disciplinas? Planteo este punto porque, por ejemplo, en mi caso específico, logré ingresar a la comunidad de investigadores de FONDECYT, mediante un proyecto realizado en conjunto con investigadores de la psicología. Así entonces, lo que acabo de comentar-aunque sea un dato personal- no es menor, pues nos habla del desafío que tenemos en el corto y mediano plazo.

Siguiendo con los lineamientos temáticos planteados, quisiera hacer hincapié en un aspecto de lo metodológico y que ocurre cuando un investigador, por ejemplo, en el campo audiovisual o del análisis de la prensa, construye su propia matriz o modelo de análisis. En este caso, el investigador tiene que asegurar una cuestión que es clave en metodología: la replicabilidad de los resultados; es decir, que otra persona usando la misma matriz que usted usó y el mismo corpus, debería llegar a los mismos resultados o, al menos, muy similares. En el caso de que no se logre, puede significar que la matriz está mal construida porque no garantiza esa replicabilidad y, en consecuencia, muchos podrán criticar o abogar falta de cientificidad en su ejecución, aunque ni siquiera estamos hablando de los niveles de confiabilidad. Pero no podemos negar entonces que frente a un reclamo de tales características tenemos un problema y el problema pasa por la formulación de un modelo de análisis y la respectiva garantía de replicabilidad, lo que nos lleva a tratar de cumplir lo siguiente: la parte informativa. Por ende, tengo que ser capaz de conceptualizar lo más rápidamente posible qué se va a entender por cada una de las categorías de esa matriz, porque eso marca mi posición. No es lo mismo definir la categoría desde la teoría de sistemas que definirla desde la economía política. Incluso lo que me interesa recalcar corresponde a lo planteado por Eliseo Verón durante la jornada de ayer. Allí se explicita que hay diversas formas de encarar lo metodológico, entonces si yo lo encaro de una forma determinada conceptualmente, se establece qué es lo que voy a entender por esa categoría, para después, continuar con hacerlo operacionalizable. Si yo no operacionalizo y describo con precisión cómo es que esta categoría definida conceptualmente de esta manera la voy a encontrar en este tipo de corpus, entonces dejo abierta la posibilidad de que una categoría se la pueda observar en un corpus de muchas maneras.

Como un dato anexo, les comento que, en la actualidad, muchos investigadores cuando utilizamos cualquier estrategia técnico-metodológica, establecemos protocolos e instrumentos. Estos son los que permiten recolectar y analizar los datos. Ambos elementos están unidos de forma permanente, puesto que cuando uno establece un instrumento y no define un protocolo está en problemas, pues entonces dicho problema lo podemos observar cuando un investigador decide aplicar encuestas, pero no define el protocolo con el cual va aplicar esa encuesta. Por ejemplo, en estos momentos, estamos llevando a cabo una investigación comparativa entre México, Argentina y Chile en el ámbito de la comunicación y la salud, para lo cual estamos trabajando con un protocolo que permita a los encuestadores de cada país la aplicación correcta del instrumento, de tal manera que la información obtenida pueda ser procesada según la modalidad de análisis establecida en el proyecto.

Por otra parte, permítanme pasar a un punto -más bien volver- que desde la perspectiva de Jesús Ibáñez (2003, 1994), estimo importante acerca del desarrollo de un tipo de investigación en comunicación. Me refiero a lo establecido por este autor, sobre el carácter histórico, comparativo y crítico de los procesos investigativos, donde se refuerza directamente la distinción que él hace entre las metodologías distributivas y estructurales. Me explico mejor. Para Ibáñez (2003, 1994), a las distributivas les interesa medir frecuencias de ocurrencia y a las estructurales les interesa buscar la comprensión de los procesos y las estructuras, porque cuando yo hago análisis de discurso desde las "historias de vida o de familia", por ejemplo, que son muy interesantes como estrategia de recolección de datos, estoy apuntando a procesos más estructurales.

Por su parte, lo que estamos haciendo en la actualidad como grupo de investigación en la Universidad de La Frontera es entender primero desde una teoría muy específica -la teoría crítica y narrativista del derecho- un objeto de estudio, así entonces analizo un corpus de sentencias penales no como hechos jurídico-judiciales, sino como relatos, por lo tanto, es factible asumir que la crisis de la justicia en el país no es una crisis del derecho o una crisis de la legislación, de modo que la solución no está en crear más leyes o modificarlas, la solución es que la gente perdió la credibilidad, la convicción en la justicia como un relato, es decir, la gente no cree en el relato de la justicia. Entonces ahí entramos a través del relato, que es la descripción que nosotros tenemos en esta investigación -que es el segundo FONDECYT-y que corresponde a la continuidad del trabajo investigativo que comenzó en 1998. Desde 2006, nos hicimos una pregunta: ¿podemos entrar desde el relato? Y la respuesta es un rotundo ¡sí podemos!, puesto que mediante un nivel narratológico, se puede acceder a una dimensión ideológica del relato-discurso y, por lo tanto, nos focalizamos en analizar esos relatos, cómo se construyen esos relatos, quienes los construyen y quienes aparecen en esos relatos. 
Por último, en el marco de estas investigaciones que estamos desarrollando en la actualidad, nos hacemos una pregunta crucial que se están haciendo varios teóricos en comunicación, en torno "al problema de lo lógico-argumental”, como lo plantea Gilberto Giménez, es decir, como vemos, el problema metodológico no es solamente un problema de tipo ideológico o un problema analítico, sino que también es un problema lógico. Entonces nosotros recuperamos un planteamiento que se está trabajando desde la lógica: analizar los discursos desde su construcción lógica. Esto se puede explicar en la medida que nosotros aplicamos un modelo de análisis que se llama Chart Method, que nos ha dado resultados interesantísimos en el plano de los resultados, porque hemos identificado a personas sentenciadas por problemas morales, ideológicos y no jurídicos. Incluso se ha podido demostrar que la línea argumental de algunas sentencias no posee una estructura lógica correcta.

En definitiva, el trabajo de investigación siempre es complejo en su desarrollo, pero no quisiera terminar sin antes vincular lo dicho con una definición de Gilberto Giménez (1982) acerca del discurso político y que me interesa muchísimo. Después de leer todo el trabajo de Gilberto Giménez, me centro en sus nociones de discurso y de argumento, él dice: "para analizar la ideología, debemos analizar los argumentos, porque los argumentos son esquemas mentales que de alguna manera registran la ideología que tienen los sujetos", entonces la matriz de análisis que se está utilizando me resulta hoy día mucho más operativa que todo el trabajo que había hecho antes de construir una matriz, porque desde los enunciados que aparecen, las frases que aparecen en los distintos discursos, de las distintas técnicas de recolección, yo llego a discursos, argumentos diría Giménez, y sobre estos argumentos finalmente tengo que establecer cómo se construyen, cómo se llega desde esa frase a establecer el argumento y cómo el investigador infiere el argumento tras la búsqueda de un proceso lógico. Eso es todo, muchas gracias.

\section{PARTICIPACIÓN DE LOS ASISTENTES}

\section{Intervención 1}

Primero que nada, quiero felicitarte por tu exposición acerca de la metodología en investigación, porque es un tema que no se discute mucho en las universidades. Bueno ustedes tienen que aplicar tales y tales modelos, pero esos modelos siempre vienen de la historia y la sociología, y nunca había visto una persona que planteara que tenemos un problema en el ámbito de la comunicación, producto de la ausencia de metodologías propias para la comunicación, lo que se traduce en un problema que tengo ahora con mi tesis. Finalmente, ¿cuál es tu propuesta, en términos sencillos, acerca del estudio del discurso?

\section{Respuesta del Dr. Carlos del Valle}

Lo primero, creo, es que tenemos que dejar de estudiar el texto como si fuera una unidad independiente, absolutamente autónoma, porque el texto está en interacción con otros textos. Lo que pasa es que detrás hay una problemática epistémica que no es menor, que es la posición inmanentista o la posición trascendentalista del texto o la metafísica que diría parafraseando la primera exposición del colega Juan Pablo Arancibia. La posición metafísica es la típica que se ha trabajado mucho tiempo, donde yo puedo hablar de un texto fuera de las posiciones que hay de este, o sea, cómo se hace referencia al texto desde un afuera trascendente. Para ser más franco, siempre se analizaba a través de una trascendencia, entonces la posición inmanente es una posición que dice "no por favor, el texto es cerrado, el texto se autoexplica y, por lo tanto, todo lo que usted tiene que encontrar tiene que derivarse de lo que está en el texto”. Esa posición que también ha llevado una posición teológica sistemática, es un enfoque de decir "si usted analiza un texto, tiene que cumplir con tres principios: solo el texto, primero el texto y todo el texto (sola, tota y prima scriptura)". Luego vendrán otros autores que dirán "ojo, el texto está refiriéndose a un afuera”, pero es un afuera y en ese afuera encontramos muchos sentidos, entonces me gusta mucho la definición de Gonzalo Abril (2009), cuando habla del "exo-inmanentismo crítico". Desde mi punto de vista, es la discusión actual en nuestro campo, en la medida que la discusión tiene que ver básicamente con el texto y su relación con otros. Muy bien, muchísimas gracias de nuevo.

\section{BIBLIOGRAFÍA}

Abril, G. (2009). “'Se puede hacer semiótica y no morir de inmanentismo?”, I/C - Revista Científica de Información y Comunicación, 6, pp. 127-147.

Bourdieu, P. (2003). Homo academicus, Buenos Aires: Siglo XXI. Giménez, G. (1982): Poder, Estado y Discurso, México, UNAM/ Instituto de Investigaciones Jurídicas (3a. edición, 1989).

Ibáñez, J. (2003). Más allá de la sociología. El grupo de discusión: técnica y crítica, Madrid, Siglo Veintiuno de España Editores.

Ibáñez, J. (1994). Por una sociología de la vida cotidiana, Madrid, Siglo XXI.

Jameson, F. (1989). Documentos de cultura, documentos de barbarie: La narrativa como acto socialmente simbólico. Madrid, Visor Distribuciones S. A.

Martin Serrano, M. (2004). La producción social de comunicación, Madrid, Alianza Editorial.

Piñuel, J. L. (2002). “Epistemología, metodología y técnicas del análisis de contenido”, en Estudios de sociolingüística: Lenguas, sociedades e culturas, Vol. 3, No 1, pp. 1-42 